\title{
The Discrimination Method of Regional Traffic Status under Earthquake
}

\author{
Chen Hong ${ }^{1, a}$, Yu Feng ${ }^{2, b}$, Zhou Hu-xing $^{3, c}$ \\ ${ }^{1}$ College of Computer and software, Nanjing Institute of Industry Technology, Nanjing, 210046, \\ China \\ ${ }^{2}$ College of Electric and Electronic Information, Changchun Architecture and Civil Engineering \\ College, 130000, Changchun, China \\ ${ }^{3}$ College of Transportation, Jilin University, 130022, Changchun, China \\ a chenh@niit.edu.cn, ${ }^{\text {b }}$ Professor yu@sina.com, c zhouhuxing@gmail.com
}

Keywords: engineering of communications and transportation; earthquake; traffic capacity; traffic status discrimination

\begin{abstract}
On the basis of existing traffic status discrimination under normal conditions, considering the influence of earthquake disaster on road capacity, real-time discrimination methods of section and intersection under earthquake was proposed. Through considering factor such as road level, section capacity and section length, determined traffic status weights of sections and intersections, achieved the real-time discrimination of regional traffic status. The method was verified using simulation road network. The results showed that under earthquake the method has good performance in aspects of accuracy and prediction time.
\end{abstract}

\section{Introduction}

At present, research of road traffic abnormal state identification under normal circumstances is relatively mature. According to different forms of Information Obtaining, divide road traffic abnormal state identifications under normal circumstances into three categories: traffic state identifications based on information from detectors in road ${ }^{[1,2]}$, traffic state identifications based on information from detectors in car ${ }^{[3]}$ and traffic state identifications based on different information fusion ${ }^{[4]}$. However, when the earthquake occurs, research of road traffic abnormal state identification is relatively less, results of regional traffic state discrimination is the basis of switching on emergency traffic organization plan, is important to rescue disaster victims and transport living materials. In this paper, propose regional traffic state identification method based on weight of road network elements.

The impact of earthquake on transportation system is regional. Usually, it spreads from source to all-round, the extent of damage decreases with the increase of distance from earthquake source. Traffic flow may be cut off by serious damaged road or collapsed roadside buildings. At this point, the road capacity is zero and section will have to plug. Another situation is that although road is damaged but road capacity is not decrease to zero, regional traffic state discrimination in this case is the study focus.

\section{Road capacity estimation after earthquake}

After earthquake, the first data we got is destruction of road system. It reflects in three aspects: (1) Road destruction, including pavement crack, muster or frost boil, (2) Bridge, culverts and other transportation structures destruction, when these structures were destruct, road capacity would be affected greatly, and (3) Street building and road ancillary facilities collapse ${ }^{[5]}$.

The existing road network includes sections and intersections, this paper views bridges including viaducts as separate part. When calculate road capacity after earthquake, in addition to considering road destruction itself, also consider collapse of street buildings and ancillary facilities, because impacts of street buildings collapse to bridge is small, only consider bridge itself destruction to road traffic system capacity. 
For the damage of road and bridge itself by earthquake, use Eq. (1) and Eq. (2) to calculate:

$$
\overline{\text { ind }_{i}}=\left[\prod_{j=1} X_{i j}\right] * 0.2-0.1
$$

Where $\overline{i n d_{i}}$ is the average earthquake index of section $i, X_{i j}$ is quantify value of earthquake damage factor $j$ in section $i$, specific damage factors and corresponding quantization values refer in paper GIS-based earthquake disaster prediction and connectivity analysis for urban road transit system. According to the getting mean earthquake damage index, contrast with road disaster grade table and road capacity probability table in referred paper, we get road approximate capacity after earthquake.

$$
y_{i}=c_{0} * \prod_{j=1}^{n} \prod_{k=1}^{l} c_{j k}^{x_{j k}}(i=1,2, \ldots . ., m ; j=1,2, \ldots . ., n ; k=1,2, \ldots \ldots, l)
$$

Where $m$ is number of samples, $n$ is number of indexes affecting earthquake damage on bridge, $l$ is classification number of index $j, y_{i}$ is earthquake damage index of bridge $i, x_{i j k}$ value is 0 or 1 . The specific earthquake damage indexes and coefficients refer paper prediction method of the earthquake disaster on highway bridge.

When calculate impact of street construction collapse on traffic capacity, using method referred in paper simulation on accessibility of post-earthquake urban transportation system, but re-divide street building type into masonry structure, frame bottom structure and concrete structure. At the same time, consider the fallout of destructed building is closely related to building height, when calculation we give 0.6 to facade area weight and 0.4 to surface area, as Eq. (3):

$$
A_{m}=0.6 * B_{l}+0.4 * A_{l}
$$

\section{Traffic status identification of section after earthquake}

After getting road capacity, we can conclude that if the capacity is not zero and there have cars travelling on road, we could use traffic parameters data to identify traffic states. Compared with traffic flow in intersection, section's traffic is more stable. Therefore, study traffic states identification on section first.

According to Pipes-Munjal model which reflects that speed is inversely proportional to traffic density, analyze actual traffic conditions on section and then use model reflecting. Different density corresponds distinct function between speed and density, the relationship is as Eq. (4):

$$
v=v_{f}\left(1-k / k_{j}\right)^{n}
$$

Where $v_{f}$ is free-flow speed. In general, it is road design speed $(\mathrm{km} / \mathrm{h}) . k_{j}$ is jamming density (vehicles/km).

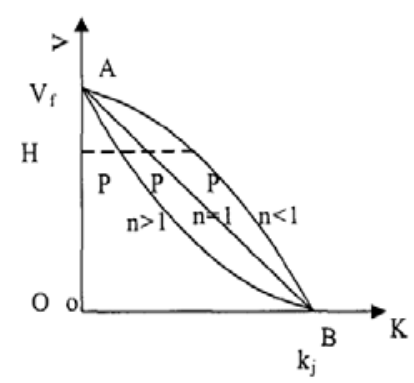

Fig.1 Speed-density diagram

From Fig. 1, we know that in point A, vehicles travel in free-flow speed and traffic density is zero, in this condition, traffic freedom is maximum. As density increases, speed decreases and level of crowding increases. When reach point $\mathrm{B}$, density is jamming density, vehicle speed is zero, and traffic is block. If use speed and density describing traffic freedom, when in point $\mathrm{P}$, comparing with free-flow in point A, we use AHP area $S_{A H P}$ to express loss of freedom degree. When in point 
$\mathrm{B}$, traffic is jam, and freedom degree is zero, the loss can be express using area of AOB. If road damage is serious and reduce traffic flow to zero, regard it as point $\mathrm{B}$.

Define the congestion value indicator CV, for any sections of road network, calculate CV using Eq. (5):

$$
C V_{p}=\frac{S_{A H P}}{S_{A O B}}
$$

Assume on section $\mathrm{P}$, travel speed is $v$ and density is $k$, then:

$$
\begin{gathered}
S_{A H P}=\int_{0}^{k} v_{f}\left(1-k / k_{j}\right)^{n} d k-v k=\frac{v_{f} k_{j}}{n+1}\left[1-\left(1-k / k_{j}\right)^{n+1}\right]-k v_{f}\left(1-k / k_{j}\right)^{n} \\
S_{A O B}=\int_{0}^{k_{j}} v_{f}\left(1-k / k_{j}\right)^{n} d k=\frac{k_{j} v_{f}}{n+1} \\
C V_{p}=\frac{S_{A H P}}{S_{A O B}}=\left[1-\left(1-k / k_{j}\right)^{n+1}\right]-(n+1) \frac{k}{k_{j}}\left(1-k / k_{j}\right)^{n}=\left[1-\left(1-k / k_{j}\right)^{n}\left(1+n k / k_{j}\right)\right]
\end{gathered}
$$

Use ratio of $Q$ and $v$ to represent $k$, then use formula Eq. (4) to calculate $n$.

After getting $C V_{p}$, we can get section traffic states comparing $C V_{p}$ with pre-set threshold. Among which, fixing threshold is crucial. According to the 2008 urban traffic management evaluation index system which has average speed rank for urban trunk road on morning peak hours, as in Table 1. Considering the special requirements on traffic threshold of traffic state identification when abnormal events occur, integrate expert knowledge of traffic experts and earthquake experts, we simplify speed rank from five categories to three, due to space constraints, this article only lists speed rank of urban trunk road, as in Table 2.

\begin{tabular}{|c|c|c|c|}
\hline evaluation category & smooth & general congestion & severe congestion \\
\hline Mega-city and A city & $>25$ & {$[19,25)$} & {$[0,19)$} \\
\hline B city & $>28$ & {$[22,28)$} & {$[0,22)$} \\
\hline $\mathrm{C}$ and $\mathrm{D}$ city & $>30$ & {$[24,30)$} & {$[0,24)$} \\
\hline
\end{tabular}

Table 1 Average speed rank in urban traffic management evaluation index system $[\mathrm{km} / \mathrm{h}]$

\begin{tabular}{cccccc}
\hline Evaluation Categories & 1 & 2 & 3 & 4 & 5 \\
\hline Mega-city and A city & {$[25,30]$} & {$[22,25)$} & {$[19,22)$} & {$[16,19)$} & {$[0,16)$} \\
B city & {$[28,33]$} & {$[25,28)$} & {$[22,25)$} & {$[19,22)$} & {$[0,19)$} \\
C and D city & {$[30,35]$} & {$[27,30)$} & {$[24,27)$} & {$[21,24)$} & {$[0,21)$} \\
\hline
\end{tabular}

Table 2 Speed rank of urban trunk road $[\mathrm{km} / \mathrm{h}]$

We use Eq. (9) to calculate $k_{j}$ :

$$
Q_{\text {MAX }}=\frac{1}{4} \times v_{f} \times k_{j}
$$

Where $Q_{\text {MAX }}$ is road traffic capacity, $v_{f}$ is road design speed.

For different levels of road, according with the travel speed evaluation criteria, use Eq. (4) we can get corresponding density, then put it into Eq. (8) and obtain corresponding threshold of three states.

The intersection situation is rather special, when earthquake occurs, the road destruction may destroy collection equipments or communication system. Traffic signal control system may be damaged too. In this case, how to distinguish the traffic state in the shortest time is the focus of our research, this paper selects the distinguish method based on saturation and the key is to estimate traffic capacity of intersection after earthquake.

$$
P I_{i}=q_{i} / n c_{i}
$$


Where $q_{i}$ is the critical phase measured flow of intersection $i, n$ is the lanes number of critical phase, $c_{i}$ is the single lane capacity. The division criteria of saturation is show in Table 4 which we comprehensive consider Table 3 and experts knowledge.

Table 3 Intersection service level for large and medium cities in China

\begin{tabular}{ccccc}
\hline service level & 1 & 2 & 3 & 4 \\
\hline saturation & $<0.6$ & {$[0.6,0.75)$} & {$[0.75,0.9]$} & $>0.9$ \\
\hline
\end{tabular}

Table 4 Suggestion value of saturation in intersection after earthquake

\begin{tabular}{cccc}
\hline evaluation category & smooth & general congestion & severe congestion \\
\hline saturation & $<0.6$ & {$[0.6,0.9]$} & $>0.9$
\end{tabular}

After distinguishing traffic state of roads and intersections, need to discriminate regional road network state. The unbalanced nature of traffic state and road network structure is the key consideration factor of regional traffic state discrimination. When earthquake occurs, network element weights reflect the differences in the response of the road state between different network elements. Through establishing network element weight model, the unbalanced nature of traffic state and road network structure will be reflected in the discernment model, which can assess traffic running status reasonably.

For sections of road network, consider road level, sections capacity and sections length, according to cumulative total mileage of vehicles driving in each grade road of discrimination region, compare vehicle road kilometers of each grade road and result is the weight of main roads, secondary roads and slip roads, then according to section capacity $d_{i j}$ and section length $l_{i j}$, we get comprehensive weight of section $L_{i j}$ using Eq. (11):

$$
w_{i j}=\frac{a_{k} * d_{i j} / l_{i j}}{\sum_{i, j=1, L_{i j} \in L}^{n} a_{k} * d_{i j} / l_{i j}}
$$

Where $a_{k}$ is the weight of each grade road, $a_{1}$ represents main roads, $a_{2}$ represents secondary roads and $a_{3}$ represents slip roads.

Seek intersection weight using the weight of sections. After earthquake, the key we need to consider is the evacuation capacity of intersection. Therefore, this article regards intersection as center, then uses exit road weights calculating the intersection weight:

$$
w_{i}=\frac{1}{n} \sum w_{i j}
$$

Where $n$ is the number of intersections connected to intersection $i, j$ is the intersection $\mathrm{j}$ connected to intersection $\mathrm{i}$.

After getting $w_{i}$ and $w_{i j}$, use Eq. (13) to calculate regional congestion value:

$$
R C V=w_{i} * P I_{i}+w_{i j} * C V_{i}
$$

When intersection detectors were destroyed by earthquake, the volume data can't be collected, in this case, only use travel time delay of GPS vehicles to discriminate intersection traffic status. Considering that GPS data can offer instantaneous speed, for normal driving GPS vehicles, when instantaneous speed in each three consecutive data analysis interval time is zero and before vehicles drive to stop line every instantaneous speed is much lower than driving speed in section, we believe that vehicles start to queue from the moment $t_{1}$ of first zero speed, then record the time $t_{2}$ that vehicles pass through the stop line, using $t_{2}$ subtract $t_{1}$ we get vehicles driving time $t$ from queue 
position to stop line, the different of time ${ }^{t}$ and free-flow travel time is intersection delay time. By comparing threshold in Table 5 we get intersection traffic status.

Table 5 Intersection average delay standard of emergency accident [s]

\begin{tabular}{cccc}
\hline evaluation category & smooth & general congestion & severe congestion \\
\hline delay & $\leq 30$ & $(30,80]$ & $>80$ \\
\hline
\end{tabular}

\section{Verifying discrimination method}

\section{Simulation Road Network}

We use road network of Fig. 2 to verify regional traffic status discrimination method after earthquake.

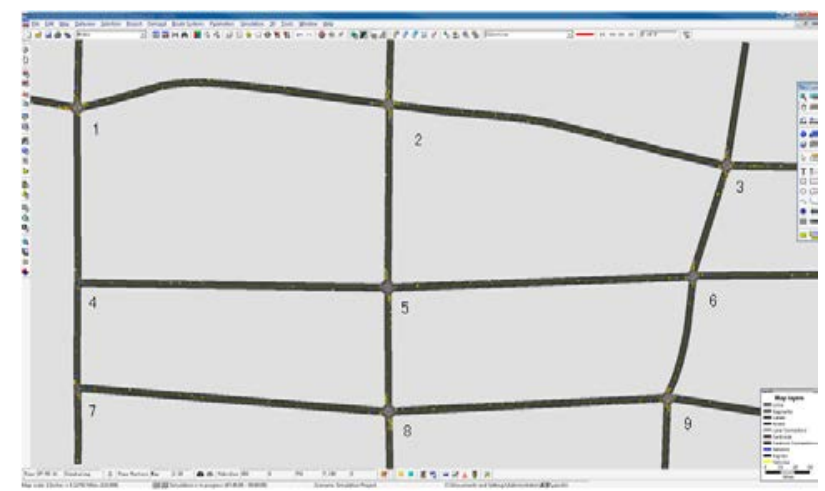

Fig.2 Regional road network simulation after earthquake

This simulation network is the central region of Dezhou City, including six main roads and nine key intersections. We take section of DongFangHong Xi Road between HuBin Zhong Avenue and DeXing Zhong Avenue for example. Firstly, calculate this section traffic capacity.

In this paper, the earthquake basic intensity is assumed as eight degree. The section subgrade soil is stiff soil, site category is class III, subgrade failure degree is slight, type is subgrade along the street, height of subgrade is $1 \mathrm{~m}$ and fortification intensity is has been fortified.

\section{Simulation Results}

Using Eq. (1), we get $\overline{i n d_{i}}$ value 0.165, compare with thresholds in table of road disaster grades description, determine that the section has suffered minor damage and traffic capacity decreases to $70 \%$ of origin capacity.

Secondly, calculate section congestion value. Use link travel speed $\mathrm{v}$ and section density $\mathrm{k}$ which are show in Table 6 to calculate $\mathrm{n}$. The value of $v_{f}$ is section design speed $50 \mathrm{~km} / \mathrm{h}$, using Eq. (9) where $Q_{M A X}$ value is $1000 \mathrm{veh} / \mathrm{h}$ we get the value of $k_{j}$ is $80 \mathrm{veh} / \mathrm{km} / \mathrm{lane}$. Through fitting calculation the value of $n$ we got is 3.6.

Table 6 Parameter data of section from simulation network [km/h] [veh/km/lane]

\begin{tabular}{ccccccc}
\hline & $8: 05$ & $8: 10$ & $8: 15$ & $8: 20$ & $8: 25$ & $8: 30$ \\
\hline section travel speed v & 17.7 & 19.0 & 17.9 & 22.4 & 17.1 & 21.7 \\
section density k & 12.4 & 9.9 & 11.6 & 9.0 & 11.2 & 9.6 \\
& $8: 35$ & $8: 40$ & $8: 45$ & $8: 50$ & $8: 55$ & $9: 00$ \\
section travel speed v & 19.2 & 21.4 & 18.9 & 20.6 & 16.6 & 20.1 \\
section density k & 11.3 & 9.4 & 11.2 & 9.8 & 12.4 & 10.6 \\
\hline
\end{tabular}

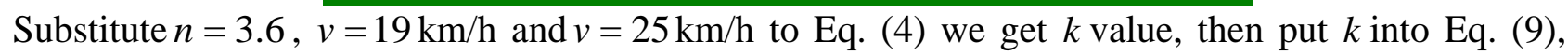
get thresholds of $C V_{p}$ are 0.1845 and 0.3501 . When put section density in Table 6 into Eq. (9) we can get section traffic status, as shown in Table 7: 
Table 7 Results of discrimination method proposed in this article

\begin{tabular}{cccccccc}
\hline & $8: 05$ & $8: 10$ & $8: 15$ & $8: 20$ & $8: 25$ & $8: 30$ \\
\hline traffic & severe & severe & severe & general & severe & general \\
status & congestion & congestion & congestion & congestion & congestion & congestion \\
& $8: 35$ & $8: 40$ & $8: 45$ & $8: 50$ & $8: 55$ & $9: 00$ \\
traffic & general & general & general & general & severe & general \\
status & congestion & congestion & congestion & congestion & congestion & congestion \\
\hline
\end{tabular}

Using vehicles travel speed got from simulation network and travel speed thresholds which are shown in Table 2, we get traffic status shown in Table 8:

Table 8 Discrimination results of contrast method

\begin{tabular}{ccccccc}
\hline & $8: 05$ & $8: 10$ & $8: 15$ & $8: 20$ & $8: 25$ & $8: 30$ \\
\hline traffic & severe & severe & severe & general & severe & general \\
status & congestion & congestion & congestion & congestion & congestion & congestion \\
& $8: 35$ & $8: 40$ & $8: 45$ & $8: 50$ & $8: 55$ & $9: 00$ \\
traffic & general & general & severe & general & severe & severe \\
status & congestion & congestion & congestion & congestion & congestion & congestion \\
\hline
\end{tabular}

\section{Results Analysis}

In order to verify discriminant rate of the method, this paper did 15 times network simulation under the same earthquake intensity by using different flow values and setting different seed number, through comparing 180 discrimination results of the two methods, we got discriminant rate is $91.6 \%$ and average discrimination time is 8.6 seconds.

\section{Conclusions}

Post-earthquake traffic state identification is the difficult of traffic fields. This paper fully considered the adverse effects of earthquakes on transportation, on the basis of section and intersection traffic state discrimination, considering factor such as road level, section capacity and section length, determined traffic status weights of sections and intersections, achieved the real-time discrimination of regional traffic status. The method was verified using simulation road network. The results showed that the method has good performance in aspects of accuracy and prediction time. This paper provides a new idea for study on post-earthquake traffic state identification.

\section{Acknowledgements}

The author expresses his sincere appreciation to the Jiangsu Province Sensor Network Engineering Open Fund No.402050612ZK201 for the financial supports of this subject.

\section{References}

[1] Stathopoulos A, Karlaftis M G. A Multivariate State Space Approach for Urban Traffic Flow Modeling and Prediction. Transportation Research Part C: Emerging Technologies, Vol. 11 (2003), p. 121-135.

[2] Guo Wei, Yao Dan-ya, Fu Yi, et al. Study on the Method for Regional Traffic Flow Feature Extraction and Traffic Status Evaluation. Journal of Highway and Transportation Research and Development, Vol. 07 (2005), p. 101-104, 114.

[3] Kerner B S, Demir C, Herrtwich R G, et al. Traffic State Detection with Floating Car Data in Road Networks. Proceedings of the 8th International IEEE Conference on Intelligent Transportation Systems, F, (2005). 
[4] Daganzo C F, Geroliminis N. An Analytical Approximation for the Macroscopic Fundamental Diagram of Urban Traffic. Transportation Research Part B: Methodological, Vol. 42 (2008), p. 771-781.

[5] Liu Jie. Study on Post-earthquake Emergency Evaluation Method of Traffic Engineering Based on GIS. Institute of Engineering Mechanics, China Earthquake Administration, (2011). 